\title{
Elevated Expression of Urokinase Plasminogen Activator in Rodent Models and Patients With Cerebral Amyloid Angiopathy
}

\author{
Marc Vervuurt \\ Radboudumc https://orcid.org/0000-0002-0444-662X \\ Xiaoyue Zhu \\ University of Rhode Island \\ Joseph Schrader \\ University of Rhode Island \\ Anna de Kort \\ Radboudumc \\ Tainá Marques \\ University Medical Center Nijmegen: Radboudumc \\ Iris Kersten \\ Radboudumc \\ Annemieke Peters van Ton \\ Radboudumc \\ Wilson Abdo \\ Radboudumc \\ Floris Schreuder \\ Radboudumc \\ Ingeborg Rasing \\ Leiden University Medical Center: Leids Universitair Medisch Centrum \\ Gisela Terwindt \\ Leiden University Medical Center: Leids Universitair Medisch Centrum \\ Marieke Wermer \\ Leiden University Medical Center: Leids Universitair Medisch Centrum
}

Steven Greenberg

Massachusetts General Hospital

Catharina Klijn

Radboudumc

Bea Kuiperij

Radboudumc

William Van Nostrand

University of Rhode Island

Marcel Verbeek ( $\sim$ marcel.verbeek@radboudumc.nl)

Radboud University Medical Center https://orcid.org/0000-0003-4635-7876

\section{Research article}

Keywords: cerebral amyloid angiopathy, urokinase plasminogen activator, biomarker, amyloid beta, cerebrospinal fluid

Posted Date: October 26th, 2021

DOI: https://doi.org/10.21203/rs.3.rs-990673/v1 
License: (c) (1) This work is licensed under a Creative Commons Attribution 4.0 International License. Read Full License

Version of Record: A version of this preprint was published at Neuropathology and Applied Neurobiology on March 9th, 2022. See the published version at https://doi.org/10.1111/nan.12804. 


\section{Abstract}

Objective: To study the association of urokinase plasminogen activator (UPA) with development and progression of cerebral amyloid angiopathy (CAA) in a rat model for CAA type-I ( $\mathrm{rTg}-\mathrm{DI}$ ) and in patients with sporadic (sCAA) and Dutch-type hereditary CAA (D-CAA). Additionally, to investigate the potential of uPA to serve as a diagnostic biomarker of CAA in cerebrospinal fluid (CSF) from patients with sCAA and D-CAA.

Methods: We studied the expression of uPA mRNA by qPCR and co-localization of uPA with amyloid- $\beta$ (A $\beta$ ) using immunohistochemistry in the cerebral vasculature of rTg-DI rats compared to wild type (WT) rats and in an sCAA patient and control subject using immunohistochemistry. CSF levels of uPA were measured in rTg-DI and WT rats and in two separate cohorts of sCAA and D-CAA patients and controls, using enzyme-linked immunosorbent assays (ELISA).

Results: The presence of uPA was clearly detected in the cerebral vasculature of rTg-DI rats and an sCAA patient, but not in WT rats or a non-CAA human control. uPA expression was highly co-localized with microvascular A $\beta$ deposits. In rTg-DI rats, uPA mRNA expression was highly elevated at 3 months of age (coinciding with the emergence of microvascular CAA deposition) and sustained up to 12 months of age (with severe microvascular CAA deposition) compared to WT rats. CSF uPA levels were elevated in rTg-DI rats compared to WT rats $(p=0.03)$, and in two separate, independent groups of sCAA patients compared to controls (after adjustment for age of subjects, $p=0.05$ and $p=0.03$ ). No differences in CSF uPA levels were found between asymptomatic and symptomatic D-CAA patients and their respective controls (after age-adjustment, $p=0.09$ and $p=0.44$ ).

Conclusion: uPA expression appears linked to the onset of CAA in rTg-DI rats. Increased cerebrovascular expression of uPA in CAA correlates with increased quantities of CSF uPA in rTg-DI rats and human CAA patients, suggesting that uPA could serve as a biomarker for CAA. No differential expression levels of UPA in CSF of (a)symptomatic D-CAA patients were discovered. These studies also further support the use of rTg-DI rat models as a useful preclinical model for the study of human CAA type-I.

\section{Background}

Cerebral amyloid angiopathy (CAA) is a highly prevalent form of cerebral small vessel disease characterized by the progressive accumulation of amyloid- $\beta(A \beta)$ peptide species in the vasculature of the brain. CAA can occur across various calibers of cerebral vessels (small arteries, arterioles, capillaries, veins and venules) [1]. A $\beta$ deposition occurring in arteries, arterioles, as well as capillaries is designated as CAA type-I, while amyloid deposition solely occurring in arteries and arterioles, but not in cerebral capillaries is referred to as CAA Type-II [2]. Due to the underlying amyloidotic pathology, CAA often coincides with Alzheimer's disease (AD), a common form of dementia in which $A \beta$ peptides are deposited in the parenchyma of the brain in the form of senile plaques [3]. As a clinical syndrome, CAA can cause vascular cognitive impairment and dementia and is an important risk factor for the development of lobar haemorrhage [4].

CAA can occur as a sporadic entity (SCAA), but mutations in the APP gene can also predispose to the development of CAA. One of the most common autosomal mutations predisposing for this hereditary form of CAA is the so-called Dutch variant of CAA (DCAA), caused by the mutation E693Q in the APP gene [5]. D-CAA causes symptoms similar to sCAA (including cognitive deterioration and repeated haemorrhages), although the clinical onset is approximately 20 years earlier and disease course is usually more aggressive. Because of the similarities in $A \beta$ composition and clinical symptoms, D-CAA can be considered a model for SCAA [6-8].

Since a brain biopsy is rarely performed for definitive CAA identification, the clinical diagnosis of sCAA is highly dependent on magnetic resonance imaging (MRI) biomarkers to provide either a probable or a possible clinical diagnosis. These MRI-biomarkers form the basis for the modified Boston Criteria to diagnose CAA and include lobar (micro-)haemorrhages and cortical superficial siderosis [9]. However, these criteria are not optimal: The imaging biomarkers often appear only after significant progression of the disease and reflect mid to end-stages of CAA, especially in the case of cerebral (micro)bleeds [10]. In addition, presence of microbleeds in non-lobar locations precludes the diagnosis of CAA, whereas CAA and arterioloslerotic small vessel disease may in fact co-exist. 
Timely CAA diagnosis could significantly benefit from novel specific biomarkers at earlier disease stages. Clinical relevance of a timely and correct CAA diagnosis is reflected in the dilemma of deciding to treat, or not to treat, atrial fibrillation with (oral) anticoagulants. Treatment with anticoagulants lowers the odds of a patient suffering of ischemic stroke, whereas this increases the odds of inducing CAA-induced intracerebral haemorrhages [11].

Proteins present in cerebrospinal fluid (CSF) may offer an excellent source of such novel biomarkers, given the close contact between the CSF and brain interstitial fluid, and thus, reflecting the pathological processes occurring in the brain and its blood vessels. For example, concentrations of $A \beta 42$ and $A \beta 40$ peptides are lowered in the CSF of CAA patients, when compared with control subjects and AD patients [12-14]. Also, $A \beta$ and in the CSF of a transgenic rodent model of CAA when compared with control rats [15]. However, given the substantial overlap of CSF A 42 and A 40 levels between CAA and AD patients, other biomarkers more specific to CAA, either as a single marker, or a combination of markers, would facilitate more accurate and timely diagnosis of the disease.

A potential candidate biomarker for CAA is urokinase plasminogen activator (UPA), a serine-proteinase that cleaves plasminogen into plasmin in the fibrinolytic pathway. UPA, and its tissue counterpart, tissue plasminogen activator (tPA), have been linked to (vascular) amyloidosis occurring in AD and CAA [16-19]. Also, fibrillar A $\beta$ accumulation can induce (over)expression of uPA, and tPA in vitro in cultured rat neurons [20]. Additionally, it has been reported that A $\beta$ peptides are cytotoxic to smooth muscle cells and pericytes, and induce expression of the proteolytically active uPA (and its receptor) by cerebral vascular smooth muscle and endothelial cells [21-23]. Lastly, uPA and other components of the plasmin system (including plasmin and tPA), have been reported as mediators of $A \beta$ degradation $[24,25]$.This myriad of relationships between vascular $A \beta$-amyloidosis and uPA implies a potential role for UPA in CAA pathophysiology, supporting its potential as a diagnostic biomarker for CAA.

Here, we analyze the potential of UPA in CSF to support the diagnosis of CAA. First, we researched amyloid deposition in brain tissue of a transgenic rat model of CAA (rTg-DI) compared to wild-type (WT) rodent controls and of a CAA patient compared to a human control subject. Additionally, we researched the extent to which uPA mRNA is produced by cerebral vessels in rTg-DI rat models at different ages, in comparison to cerebrovascular expression in WT rodent controls. Lastly, we determined uPA levels in CSF of both rTg-DI rodent models and WT rodents, and in human CAA patients and controls in two separate cohorts and researched their diagnostic performance, to determine whether CSF UPA can function as a diagnostic biomarker for CAA.

\section{Methods}

\section{rTg-DI transgenic rat model of CAA type-I}

The rTg-DI rat model expresses low levels of human amyloid precursor protein (APP) harboring the E693Q/D694N Dutch/lowa familial CAA mutations, under the control of the neuronal-specific Thy1.2 promoter, as well as induced overexpression through double K670N/M671L Swedish APP-mutations [26]. Cerebral vascular A 3 fibril deposition starts at approximately 3 months of age throughout the cortex, hippocampus and thalamus and progresses dramatically with advancing age. Non-transgenic, littermates served as wild-type (WT) control rats. For this study, heterozygous rTg-DI and WT rats were used and analyzed at 3 months (emergence of CAA) and 12 months (late stage of CAA). All animal model work was performed in accordance with the United States Public Health Service's Policy on Humane Care and Use of Laboratory Animals and was approved by the University of Rhode Island Institutional Animal Care and Use Committee (IACUC).

\section{Brain tissue collection \& preparation}

The rTg-DI and WT rats were sacrificed at the designated ages and perfused with cold phosphate buffered saline (PBS).

Forebrains were subsequently extracted and dissected through the midsagittal plane. One hemisphere was snap-frozen in optimal cutting temperature medium (OCT 4585, Fisher Healthcare, United Kingdom) directly. The other hemisphere was snap-frozen in dry ice and used for molecular measures described below. Fresh frozen human cortical brain tissue from a 71 years old male sCAA patient and a 70 years old male control patient were obtained from the University of California Alzheimer's Disease Research Center (UCI-ADRC) and the Institute for Memory Impairments and Neurological Disorders.

\section{Immunohistochemistry analysis}

Page $4 / 16$ 
Sections of $\mathrm{rTg}$-DI and control rat hemispheres were cut from fresh frozen tissues in the sagittal plane at $14 \mu \mathrm{m}$ thickness using a cryostat (Leica, Buffalo Grove, IL, USA) and placed on slides. Tissue sections were blocked in Superblock blocking buffer (37518, ThermoFisher, Waltham, MA, USA) containing $0.3 \%$ Triton X-100 at room temperature for 30 min and incubated with primary rabbit polyclonal antibody to uPA in a 1:200 dilution (American Diagnostica $\mathrm{GmbH}$, Pfungstadt, Germany). The primary antibody was detected with Alexa Fluorescent 594-conjugated secondary antibody in a 1:1000 dilution (Molecular Probes Europe BV, Leiden, Netherlands). Deposits of fibrillar vascular amyloid were detected by staining with thioflavin S (ThS; \#123H0598, Sigma-Aldrich, Saint Louis, MO, USA). Immunohistological images were captured on a KEYENCE BZ-X710 fluorescence microscope and analyzed with BZ-X Analyzer software (Keyence, Itasca, IL, USA).

\section{Quantitative real-time reverse transcriptase polymerase chain reaction}

Rat hemispheres were homogenized using Trizol (162711, Invitrogen, Carlsbad, CA, USA), after which total RNA was extracted according to manufacturer's protocol using the Direct-zol RNA MiniPrep kit (ZRC200796, ZYMO research, Irvine, CA, USA). Total RNA levels were determined using Nanodrop (Nanodrop one, Thermo Scientific, Waltham, MA, USA). This was followed by cDNA generation using the High Capacity cDNA Reverse Transcription Kit (00289994, Applied Biosystems, Waltham, MA, USA) according to the protocol supplied by the manufacturer. Quantitative polymerase chain reaction (qPCR) was performed in a Step One Plus Real-time PCR employing the Taqman primers Rn00565261_m1 (UPA) and Rn00667869_m1(Actin-beta) as an internal control system (Applied Biosystems, Waltham, MA, USA). Rat tissues of rTg-DI/control rats were analyzed using qPCR at 3 months $(n=$ 7/4), 6 months $(n=6 / 5)$ and 12 months $(n=5 / 5)$ respectively.

\section{Rat CSF collection and preparation}

Rat CSF was acquired from the cisterna magna of both rTg-DI and WT rats at 3 months of age. Rats were anesthetized deeply through isoflurane inhalation, and were subsequently mounted on a stereotaxic unit. An incision was made along the midline of the head and neck, starting between the ears and ending at about $2.5 \mathrm{~cm}$ caudally. The fascia was pulled back, and muscles were dissected, which opens up the atlanto-occipital membrane. A small incision was made along the membrane midline and its underlying dura, aided by a surgical microscope. CSF was sampled through the slit in the dura using a fine-tipped pipette. Samples were aliquoted into sterile, polypropylene (PP) tubes, and were subsequently frozen at $-80^{\circ} \mathrm{C}$. This procedure makes it possible to acquire an average of 120-150 $\mu \mathrm{L}$ CSF per rat.

\section{Rat CSF uPA ELISA}

The levels of uPA in CSF of both rTg-DI and WT rats (3 months of age, $n=3$ per group) were measured in triplicate using a Rat uPA ELISA kit (MyBioSource, San Diego, CA, USA). ELISA was performed according to manufacturer's instructions. Rodent CSF samples were diluted five times with PBS prior to analysis.

\section{Human subject inclusion and sample collection}

We analyzed the diagnostic performance of UPA as a novel biomarker for SCAA in two, separate groups, a discovery and a validation cohort.

In the discovery cohort, we obtained human CSF samples of SCAA patients $(n=27)$ and control subjects $(n=40)$ from the Radboud University Medical Center (Radboudumc; Nijmegen, the Netherlands). sCAA patients were diagnosed on the basis of the modified Boston Criteria, and were classified as either probable CAA $(n=21)$, possible CAA $(n=2)$, or CAA presenting with mixedlocation cerebral haemorrhages (lobar and deep haemorrhages/microbleeds, $n=4)$ [9]. The control group $(n=40)$ was composed from two subgroups: the majority of subjects $(n=30)$ underwent a lumbar puncture as part of the diagnostic workup of neurologic symptoms or to exclude central nervous system involvement of a systemic disease. They neither had the suspected neurological disease, nor a neurodegenerative disease, known cognitive impairment, sepsis, a recent stroke (< 6 months), or a malignancy in the central nervous system. Additional inclusion criteria were the availability of a sufficient amount of CSF and a normal composition of the CSF for a number of routine parameters (e.g. cell count, blood pigments, total protein, glucose, lactate, oligoclonal lgG bands). Additionally, a minority of CSF samples $(n=10)$ was collected from patients who underwent thoracoabdominal aortic aneurysm repair, for which they had an external lumbar drain. They did not have known cognitive impairment or recent $(<6$ months) stroke, or traumatic brain injury. Details on patient characteristics for these groups are shown in Table 1. 
Table 1

Patient characteristics and CSF parameters of the discovery group. Data is presented as median (IQR). pvalues for CSF parameters are shown in two scenarios: (1) unadjusted and (2) adjusted for age of subjects. * $p \leq 0.05 ; * \star *<<0,001$; non-significant (ns) $p>0.05$

\begin{tabular}{|lllll|}
\hline & sCAA & Controls & p-value & \\
\hline Demographics & & & \\
\hline Number of patients & 27 & 40 & - & \\
\hline Age $(\mathrm{y})$ & $73.4(67.2-77.1)$ & $64.2(56.1-69.8)$ & $\mathrm{p}<0.001(* \star *)$ & \\
\hline Sex, M/F (\% male) & $19 / 8(70 \%)$ & $28 / 12(70 \%)$ & $\mathrm{p}=0.97(\mathrm{~ns})$ & \\
\hline CSF parameters & & & Unadjusted & Adjusted (Age) \\
\hline uPA $(\mathrm{pg} / \mathrm{mL})$ & $92.5(79.8-109.6)$ & $68.4(56.6-78.4)$ & $\mathrm{p}<0.001(* \star *)$ & $\mathrm{p}=0.05(*)$ \\
\hline Aß40 $(\mathrm{ng} / \mathrm{mL})$ & $7.58(4.92-9.28)$ & $9.08(6.14-12.53)$ & $\mathrm{p}=0.06(\mathrm{~ns})$ & $\mathrm{p}=0.03(*)$ \\
\hline Total protein $(\mathrm{mg} / \mathrm{mL})$ & $0.94(0.85-1.05)$ & $0.84(0.75-0.97)$ & $\mathrm{p}=0.02(*)$ & $\mathrm{p}=0.16(\mathrm{~ns})$ \\
\hline
\end{tabular}

For validation of our initial findings, we studied additional samples from SCAA, D-CAA (both symptomatic and asymptomatic patients) and age-matched controls. sCAA patients were obtained from the Radboud University Medical Center $(n=12)$, the Leiden University Medical Center (LUMC, Leiden, the Netherlands) $(n=9)$ and the Massachusetts General Hospital (Boston, Massachusetts, USA) ( $n=17$ All controls $(n=40)$ were obtained from the Radboud University Medical Center. SCAA patients from all centers were diagnosed similarly as described for the discovery group. Details on patient characteristics of these groups are shown in Table 2.

Table 2

Patient characteristics and CSF parameters of the validation groups; SCAA. Data is presented as median (IQR). p-values for CSF parameters are shown in two scenarios: (1) unadjusted and (2) adjusted for age of subjects. * $\mathrm{p} \leq 0.05 ; * \star * \mathrm{p}<0,001$; non-significant (ns) $\mathrm{p}>0.05$

\begin{tabular}{|c|c|c|c|c|}
\hline & sCAA & Controls & p-value & \\
\hline \multicolumn{5}{|l|}{ Demographics } \\
\hline Number of patients & 38 & 40 & - & \\
\hline Age (y) & $67.5(60.0-75.3)$ & $71.2(64.5-74.1)$ & $\mathrm{p}=0.21(\mathrm{~ns})$ & \\
\hline Sex, M/F (\% male) & $19 / 19(50 \%)$ & $23 / 17(58 \%)$ & $p=0.52(n s)$ & \\
\hline CSF parameters & & & Unadjusted & Adjusted (Age) \\
\hline $\mathrm{uPA}(\mathrm{pg} / \mathrm{mL})$ & $100.5(85.8-127.5)$ & $91.5(73.4-114.5)$ & $p=0.08(n s)$ & $p=0.03\left({ }^{*}\right)$ \\
\hline $\mathrm{A} \beta 40$ (ng/mL) & $5.1(3.1-7.0)$ & $11.0(7.1-14.4)$ & $\mathrm{p}<0.001(* \star *)$ & $\mathrm{p}<0.001(\star \star \star)$ \\
\hline Total protein $(\mathrm{mg} / \mathrm{mL})$ & $0.89(0.84-1.02)$ & $0.89(0.81-0.98)$ & $p=0.57(n s)$ & $p=0.05(*)$ \\
\hline
\end{tabular}

D-CAA patients were exclusively recruited through the (outpatient) clinic of the LUMC (asymptomatic: $n=11$; symptomatic: $n=$ 12). Inclusion criteria were age $>18$ years and a proven APP mutation, or a medical history of one or multiple lobar ICHs and $\geq 1$ first-degree relative with D-CAA. Symptomatic D-CAA was defined by a history of at least one symptomatic ICH. Age-matched control groups were constructed for the asymptomatic and symptomatic D-CAA groups ( $n=22 ; n=28$ respectively). Details on patient characteristics in D-CAA groups and respective controls are shown in Table 3. 
Table 3

Patient characteristics and CSF parameters of the validation groups; D-CAA. Data is presented as median (IQR). p-values for CSF parameters are shown in two scenarios: (1) unadjusted and (2) adjusted for age of subjects.

\begin{tabular}{|c|c|c|c|c|c|c|c|c|}
\hline & $\begin{array}{l}\text { Asymptomatic } \\
\text { D-CAA }\end{array}$ & controls & $p$-value & & $\begin{array}{l}\text { Symptomatic } \\
\text { D-CAA }\end{array}$ & controls & p-value & \\
\hline \multicolumn{9}{|l|}{ Demographics } \\
\hline $\begin{array}{l}\text { Number of } \\
\text { patients }\end{array}$ & 11 & 22 & - & & 12 & 28 & - & \\
\hline Age (y) & $\begin{array}{l}38.0(31.0- \\
52.0)\end{array}$ & $\begin{array}{l}45.2 \\
(36.5- \\
51.3)\end{array}$ & $\mathrm{p}=0.61(\mathrm{~ns})$ & & $\begin{array}{l}58.5(52.8- \\
65.8)\end{array}$ & $\begin{array}{l}60.3 \\
(51.6- \\
65.9)\end{array}$ & $\mathrm{p}=0.86(\mathrm{~ns})$ & \\
\hline $\begin{array}{l}\text { Sex, M/F (\% } \\
\text { male) }\end{array}$ & $8 / 3(73 \%)$ & $\begin{array}{l}11 / 11 \\
(50 \%)\end{array}$ & $\mathrm{p}=0.213(\mathrm{~ns})$ & & $5 / 7(42 \%)$ & $\begin{array}{l}17 / 11 \\
(61 \%)\end{array}$ & $\mathrm{p}=0.27(\mathrm{~ns})$ & \\
\hline $\begin{array}{l}\text { CSF } \\
\text { parameters }\end{array}$ & & & Unadjusted & $\begin{array}{l}\text { Adjusted } \\
\text { (Age) }\end{array}$ & & & Unadjusted & $\begin{array}{l}\text { Adjusted } \\
\text { (Age) }\end{array}$ \\
\hline uPA (pg/mL) & $\begin{array}{l}90.2(74.2- \\
99.9)\end{array}$ & $\begin{array}{l}68.3 \\
(60.3- \\
88.1)\end{array}$ & $\underset{(* \star)}{p=0.01}$ & $\begin{array}{l}p=0.09 \\
\text { (ns) }\end{array}$ & $\begin{array}{l}83.4(72.4- \\
95.5)\end{array}$ & $\begin{array}{l}76.8 \\
(66.1- \\
92.7)\end{array}$ & $\begin{array}{l}p=0.46 \\
\text { (ns) }\end{array}$ & $\begin{array}{l}p=0.44 \\
\text { (ns) }\end{array}$ \\
\hline $\mathrm{A} \beta 40(\mathrm{ng} / \mathrm{mL})$ & $\begin{array}{l}2.18(1.79- \\
3.25)\end{array}$ & $\begin{array}{l}7.91 \\
(5.35- \\
10.24)\end{array}$ & $\begin{array}{l}\mathrm{p}<0.001 \\
(\star \star \star)\end{array}$ & $\begin{array}{l}\mathrm{p}< \\
0.001 \\
(\star \star \star)\end{array}$ & $\begin{array}{l}1.55(1.17- \\
2.07)\end{array}$ & $\begin{array}{l}8.72 \\
(5.94- \\
12.24)\end{array}$ & $\begin{array}{l}\mathrm{p}<0.001 \\
(\star \star \star)\end{array}$ & $\begin{array}{l}\mathrm{p}< \\
0.001 \\
(\star \star \star)\end{array}$ \\
\hline $\begin{array}{l}\text { Total protein } \\
(\mathrm{mg} / \mathrm{mL})\end{array}$ & $\begin{array}{l}0.71(0.65- \\
0.84)\end{array}$ & $\begin{array}{l}0.75 \\
(0.64- \\
0.80)\end{array}$ & $\begin{array}{l}p=0.65 \\
(n s)\end{array}$ & $\begin{array}{l}p=0.47 \\
(n s)\end{array}$ & $\begin{array}{l}0.84(0.74- \\
0.94)\end{array}$ & $\begin{array}{l}0.83 \\
(0.68- \\
0.92)\end{array}$ & $\begin{array}{l}p=0.81 \\
(n s)\end{array}$ & $\begin{array}{l}p=0.13 \\
(n s)\end{array}$ \\
\hline
\end{tabular}

The local medical ethical committees of all medical centers approved the use of patient and control CSF, and informed consent was obtained from all study subjects or their legal representatives. All patient and control subjects underwent lumbar puncture according to standard procedures. CSF was collected in polypropylene tubes and centrifuged, followed by aliquoting and storage at $-80^{\circ} \mathrm{C}$. CSF of CAA patients and controls was used to determine $A \beta 40$ levels through the Lumipulse ${ }^{\circledR} \mathrm{G} \circledast \beta-(1-40)$ chemiluminescent immunoassay (Fujirebio, Gent, Belgium). Additionally, total protein content of all CSF samples was determined using a Pierce ${ }^{\mathrm{TM}}$ BCA protein assay kit (Thermo Fisher Scientific, Waltham, MA, USA).

\section{Human CSF uPA ELISA}

Human CSF uPA levels were determined using the Human uPA Quantikine ELISA (R\&D Systems, Minneapolis, MN, USA). Undiluted human CSF was analyzed according to the manufacturer's supplied protocol. Alongside all CAA and control CSF, five pooled CSF samples were analyzed as quality controls $(\mathrm{QC})$, to check and correct for potential inter-assay variations and were used to allow for comparison between our discovery and validation cohort.

\section{Statistical analyses}

Data was analyzed using IBM SPSS Statistics Version 25.0.0.1 (IBM Corp., Armonk, NY, USA) and Graphpad Prism version 5.03 (Graphpad Software, Inc., San Diego, CA, USA).

All data shown are presented as either mean values \pm standard deviation, or median values (interquartile range; IQR) as appropriate. Statistical differences between groups were analyzed by independent t-testing or Mann Whitney U testing. Kolmogorov-Smirnov and Shapiro-Wilk tests were used to determine normality of ELISA data, whereas differences between sex were determined using the Chi-Square test of independence. Correlations between variables were determined using Pearson or Spearman correlation testing, for parametric or non-parametric data respectively. Adjustments for the influences of age of subjects on human uPA CSF levels were performed using linear regression modeling. All test results were deemed significant with a $p$-value $\leq 0.05$.

Ethical statement 
All work with animals was approved by the University of Rhode Island Institutional Animal Care and Use Committee and in accordance with the United States Public Health Service's Policy on Humane Care and Use of Laboratory Animals. Lumbar punctures and vena punctures were performed after informed consent from the patients themselves or from the patients' legal representatives. The CSF of the controls originated from a clinical diagnostic work-up or from patients that had an external lumbar drain in the context of thoracoabdominal aortic aneurism repair. sCAA patients underwent a lumbar puncture in the context of a clinical diagnostic work-up, or in the context of a cohort study on biomarkers for sCAA or D-CAA (CAVIA (no. 733050202), BIONIC (nr. 733050822), or AURORA. This study was approved by the Medical Ethics Committees Arnhem-Nijmegen and Leiden-the Hague.

\section{Results}

$\underline{\text { uPA in rTg-DI rodent models and wild-type controls }}$

We performed immunolabeling experiments to evaluate the expression of uPA in the brains of 12 months old rTg-DI rats with abundant microvascular CAA. As shown in Fig. 1A-C, we observed significant perivascular expression of uPA in cerebral capillaries harboring amyloid deposits in rTg-DI rats. In contrast, uPA expression was not appreciably detected in cerebral capillaries of WT rats (Fig. 1D).

We next evaluated uPA mRNA levels in the brain tissue of rTg-DI rats at three and twelve months of age (Fig. 2A). At 3 months of age, i.e. the age of the onset of microvascular CAA, there was a highly significant $(p<0.01) 6$-fold increase in uPA mRNA expression compared to WT rats. The increased uPA mRNA levels were sustained with a 8-fold increase in rTg-DI rats at 12 months of age compared to similarly aged WT rats $(p<0.01)$.

The early-onset uPA expression in rTg-DI rats prompted us to perform ELISA analysis to measure uPA levels in the CSF of these transgenic rats compared to WT controls at 3 months of age. Fig. 2B shows elevated levels of UPA in CSF in rTg-DI rats at 3 months of age compared to similarly aged WT rats $(39.11 \pm 3.28 \mathrm{pg} / \mathrm{mL}$ vs. $30.46 \pm 3.26 \mathrm{pg} / \mathrm{mL} ; \mathrm{p}=0.03)$.

$\underline{\text { uPA in human CAA patients and control subjects }}$

We next determined if we could confirm the increased perivascular uPA expression in our rTg-DI preclinical model of CAA in human CAA. Fig. 1E-F shows that in the presence of CAA pathology perivascular UPA expression was increased, whereas we found negligible uPA expression in vessels in a control subject lacking CAA pathology.

We next performed ELISA analysis to evaluate uPA levels in the CSF of human CAA patients and controls.

In the discovery groups (Fig. 3A; Table 1), median uPA levels in CSF (92.5 (IQR: 79.8 - 109.6) pg/mL) were higher in patients with sCAA than in control subjects $(68.4$ (IQR: $56.6-78.4) \mathrm{pg} / \mathrm{mL} ; \mathrm{p}<0.001)$. This difference was also observed when solely comparing patients with a diagnosis of probable sCAA (92.5 (IQR: 77.3 - 115.8) pg/ml) to controls. (68.4 (IQR: 56.6 - 78.4) $\mathrm{pg} / \mathrm{mL} ; \mathrm{p}<0.001)$. After adjustment for age, the difference in CSF uPA levels between sCAA patients and controls was significantly different (95\% Cl: [0.22 : 34.82], p=0.05).

In the validation groups (Fig. 3B; Table 2), median uPA levels did not differ significantly between sCAA patients (100.5 (85.8 127.5) $\mathrm{pg} / \mathrm{mL}$ ) and controls (91.5 (73.4 - 114.5) pg/mL), but did show a trend towards elevation of uPA levels in sCAA $(p=0.08)$. Adjustment of uPA levels for age resulted in a significant difference in uPA levels between sCAA patients and controls ( $95 \% \mathrm{Cl}$ : $[1.49$ : 32.7], $\mathrm{p}=0.03)$.

Comparisons between asymptomatic D-CAA patients and age-matched controls yielded a significant elevation of median CSF uPA levels in asymptomatic D-CAA patients $(90.2(74.2-99.9) \mathrm{pg} / \mathrm{mL})$ vs. age-matched controls $(68.3(60.3-88.1) \mathrm{pg} / \mathrm{mL})$, with $\mathrm{p}=0.01$. This difference was not observed when comparing symptomatic D-CAA patients with age-matched controls, with median uPA levels of $83.4(72.4-95.5) \mathrm{pg} / \mathrm{mL}$ in D-CAA and $76.8(66.1$ - 92.7) in controls $(p=0.46)$. After adjustment for age significance was lost for the differences between asymptomatic D-CAA and controls ( $95 \% \mathrm{Cl}$ : [-0.99: 14.30], $p=0.09)$ and the significance level was unaffected in symptomatic D-CAA vs. controls (95\% Cl: [-4.54: 10.21], $p=0.44$ ). Additional adjustment for total protein levels resulted in non-significant differences between asymptomatic D-CAA (95\% Cl: [-1.25 : 11.24], p=0.11) and symptomatic D-CAA 
(95\% Cl: [-4.53 : 9.96], p=0.45), and their respective controls. Lastly, we compared the uPA CSF levels between symptomatic and asymptomatic D-CAA patients. Levels between these two groups were found to be similar $(p=0.31)$.

\section{Discussion}

This study demonstrates an elevation of CSF UPA in patients suffering from CAA pathology. In two separate cohort studies, CSF uPA levels, after adjustment for age, were significantly elevated in SCAA patients, when compared with respective controls. In contrast, no elevation of uPA levels was observed after adjustment for age in (a)symptomatic D-CAA patients, compared to their respective age-matched controls. Perivascular uPA in amyloid-affected cerebral vessels was overexpressed in rTg-DI rats and a sCAA patient. Moreover, uPA (mRNA) levels were increased in CSF from the rTg-DI CAA rodent model.

Earlier studies have demonstrated a decreased of uPA levels in plasma, but not CSF, in mild-cognitive impairment (MCl) patients with decreased $A \beta 42 / A \beta 40$ ratios (indicative of amyloid pathology) compared to controls [27]. Similarly, no differences in the CSF concentrations of plasminogen, tPA or plasminogen activator inhibitor-1 between AD patients and controls were discovered. Moreover, in this study zymography analysis in CSF revealed no difference in TPA activity between AD and controls, whereas uPA activity could not be detected in CSF of either AD or control cases [28]. However, in one other study, increased levels of CSF tPA were observed in $A D$ vs. patients with subjective cognitive impairment, but not in $A D$ vs. $M C l$ patients [29]. In summary, these studies show that UPA as an influential factor in CAA is barely being researched and that UPA CSF levels are not increased in AD, whereas inconsistent results were obtained for tPA levels in AD. Despite the partial overlap in pathology of AD and CAA, elevated levels of UPA in CSF seem to be restricted to CAA. Future studies should reveal if uPA levels are increased in AD patients with severe CAA compared to AD cases without CAA [3].

Endothelial cells are a major source of vascular uPA expression, thereby suspected of major contributors to the activation of the plasminogen activation system, through the conversion of plasminogen into plasmin [30,31]. Because of this role in plasmin production, uPA and other components of the plasminogen activation system, are often associated with tissue remodeling processes, like cellular migration and metastasis, hemostasis, fibrinolysis or angiogenesis [32]. Earlier studies have shown elevated expression of both uPA and its receptor, UPAR, after stimulation of human smooth muscle cells with A [23]. Whereas the colocalization of uPA with A $\beta$ in CAA patients and rTg-DI models we describe here has not been described before, tPA, the tissuecounterpart of UPA, has been found to colocalize with AB in the cerebral vasculature of Tg2576 mice, an AD mouse model [33]. The co-localization of tPA and A $\beta$ may be explained by the observations that both TPA and plasminogen bind to lysine-rich structures which are present both in fribrin and in $A \beta[19]$.

Elevated cerebrovascular expression of uPA may stimulate the localized conversion of plasminogen to plasmin, which in a compensatory feedback-mechanism might directly decrease the vascular amyloid load through plasmin, a known A $\beta$-cleaver [20, $25,33-35]$. This proposed mechanism for the plasminogen activation system in cleaving (vascular) A $\beta$ may also take place in senile plaques [36-43]. Whereas the uPA-induced elevated plasmin level may thus decrease vascular amyloid load, elevated plasmin levels may also negatively affect the integrity of the extracellular matrix (ECM) of affected tissues. This secondary effect could occur either directly, through plasmin-mediated focal degradation of matrix proteins, or indirectly, through the uPA- or plasmin-mediated activation of other proteolytic factors, such as the matrix metalloproteinases MMP-2, MMP-3, MMP-9, MMP-12 and MMP-13 [44-47]. Degradation of the ECM directly compromises the integrity of the vascular wall and potentially contributes to the development of cerebral (micro)haemorrhages associated with CAA.

Specific protein biomarkers in CSF may discriminate between CAA patients, AD patients and/or control subjects: e.g. decreased CSF A 40 discriminates sCAA from controls $[12,14,48]$. These previous studies also revealed decreased CSF A 42 levels in sCAA patients in comparison with controls. Also, in D-CAA decreased CSF A 340 and AB42 levels were found in asymptomatic and symptomatic mutation carriers in comparison with age-matched controls [49]. However, CSF A 42 levels were similarly decreased in $A D$ patients as well, limiting the specificity of CSF AB42 as a biomarker for CAA. Other protein biomarkers have been associated with sCAA, but again, with limited specificity (e.g. CSF Apolipoprotein D) [50].

We observed no significant differences in CSF uPA levels between symptomatic D-CAA patients (either unadjusted or when adjusted for age of subjects) and asymptomatic D-CAA patients (borderline insignificant, after adjustment for age of subjects). We 
did however observe a difference in unadjusted CSF uPA levels between asymptomatic D-CAA patients and controls. This could be induced by age-effects (as the difference is not present in our age-adjusted model), but could also have a physiological cause. We can only speculate on the origins of this difference, but it is not unlikely that progessive deposition of $A \beta$ induces an elevation of UPA expression and secretion in SCAA and asymptomatic D-CAA patients. The loss of uPA-producing endothelial cells following cerebral vessel rupture, may lead to a normalization of UPA expression in symptomatic D-CAA patients relative to asymptomatic DCAA patients. Longitudinal studies on changes in CSF uPA levels in SCAA and D-CAA patients may yield more insight into these possible sequence of events .

Several limitations apply to our study. First, the number of rats included in the CSF study was relatively small. However, the homogeneity of this particular transgenic rat population reduces the influence of confounding factors. Second, despite the observation that our clinical cohorts were of relatively moderate sizes, these sizes are comparable to those in previously peerreviewed CAA studies, which have been proven capable of producing reliable results $[14,50]$. Third, it would have been interesting to also include patients suffering from $A D$ (with vs. without evidence of $C A A$ ) and hypertensive vasculopathy as a different cause of haemorrhages in this study to address the specificity of our findings. Unfortunately these samples were not available to us, but including them in future studies could offer novel opportunities for research about the specificity of this biomarker.

Strengths of our study include the use of a unique animal model of CAA. This animal model not only recapitulates many of the characteristics of human CAA, but also allows for relatively easy collection of CSF for biomarker studies. Furthermore, the cohorts of all types of CAA patients (sporadic, asymptomatic D-CAA and symptomatic D-CAA) and controls (well-phenotyped using MRI, CSF and clinical data) are unique and a valuable source for studies of novel biomarkers of both sporadic, as well as hereditary CAA. Finally, the similarities between the animal and human findings strengthen the translational capacity of the rat model to study the pathogenesis of CAA and vice versa.

In conclusion, our findings show a multifaceted relationship between uPA and CAA pathophysiology, in both the rTg-DI CAA model and in CAA patients. Increased cerebrovascular uPA expression was observed in both rat and human CAA tissue; elevated CSF uPA levels were found in both rTg-DI CAA model rats and sCAA patients (after adjustments for age). Symptomatic D-CAA patients did not show robust differences when compared to control groups, but CSF uPA levels trended towards an elevation in asymptomatic D-CAA patients vs. controls. The association of UPA with CAA provides new insights in pathophysiological processes in both sporadic and hereditary CAA and could serve as a vascular biomarker for CAA in conjunction with other CAA-specific biomarkers.

\section{Abbreviations}

$A \beta 40=$ amyloid $-\beta 40 ; A \beta 42=$ amyloid $-\beta 42 ; A D=$ Alzheimer's disease $; A P P=$ amyloid precursor protein; $A U C=$ area under the curve; $\mathrm{BCA}=$ bicinchoninic acid assay; $\mathrm{CAA}=$ cerebral amyloid angiopathy; $\mathrm{Cl}=$ confidence interval; $\mathrm{CMB}=$ cerebral microbleed; $\mathrm{CNS}=$ central nervous system; CSF = cerebrospinal fluid; D-CAA = Dutch-type hereditary CAA; ECM = extracellular matrix; HSMC = human smooth muscle cell; $\mathrm{MR}(\mathrm{I})$ = magnetic resonance (imaging); $\mathrm{PBS}=$ phosphate buffered saline; $\mathrm{PP}=$ polypropylene; $\mathrm{qPCR}=$ quantitative polymerase chain reaction; RNAseq = ribonucleic acid sequencing; ROC = receiver operating characteristic; rTg-DI = transgenic rat model with APPE693Q/D694N Dutch/lowa mutations; ThS = thioflavin S; tPA = tissue plasminogen activator; $\mathrm{UPA}(\mathrm{R})=$ urokinase plasminogen activator (receptor); $\mathrm{WT}$ = wild type

\section{Declarations}

Author Contributions

Van Nostrand and Verbeek designed the study. Zhu and Schrader were responsible for the data collection and analysis of the animal model. de Kort, Schreuder, Klijn, Peters van Ton, Abdo, Greenberg, Wermer, Rasing and Terwindt were responsible for recruiting patients and then collection of patient data. Vervuurt, Marques, Kersten and Kuiperij performed the data collection and analysis in human subjects. Vervuurt, Kuiperij, Verbeek, and Van Nostrand interpreted the data. Vervuurt wrote the manuscript draft. All authors reviewed and contributed to the manuscript and approved the final version.

Availability of data and materials

Page $10 / 16$ 
The datasets used and/or analysed during the current study are available from the corresponding author on reasonable request.

Funding

Supported by the National Institutes of Health, USA [grant number 5R01NS104147-02] , the BIONIC project (ZonMW, nr. 733050822), and the Selfridges Group Foundation (nr. NR170024). The BIONIC project is part of 'Memorabel', the research and innovation programme for dementia, as part of the Dutch national 'Deltaplan for Dementia': zonmw.nl/dementiaresearch"

Disclosures

Vervuurt, Zhu, Schrader, de Kort, Marques, Kersten, Peters van Ton, Abdo, Rasing, Terwindt, Greenberg, Kuiperij, Verbeek, and Van Nostrand report no relevant disclosures for this manuscript.

Prof. Klijn is is supported by a clinical established investigator grant from the Dutch Heart Foundation (2012 T077) and an ASPASIA grant from the Netherlands Organization for Health Research and Development (ZonMw; 015008048). Dr. F. Schreuder is supported by a senior clinical scientist grant from the Dutch Heart Foundation (2019T060).

Prof Wermer is is supported by a clinical established investigator grant from the Dutch Heart Foundation (2016 T086) and a VIDI (91717337) and ASPASIA grant from the Netherlands Organization for Health Research and Development.

\section{References}

1. Sporadic cerebral amyloid angiopathy revisited: recent insights into pathophysiology and clinical spectrum. Journal of Neurology, Neurosurgery \& Psychiatry 2012, 83:124-137.

2. Thal DR, Ghebremedhin E, Rub U, Yamaguchi H, Del Tredici K, Braak H. Two types of sporadic cerebral amyloid angiopathy. J Neuropathol Exp Neurol. 2002;61:282-93.

3. Greenberg SM, Bacskai BJ, Hernandez-Guillamon M, Pruzin J, Sperling R, van Veluw SJ. Cerebral amyloid angiopathy and Alzheimer disease - one peptide, two pathways. Nature Reviews Neurology. 2020;16:30-42.

4. Smith EE, Eichler F. Cerebral Amyloid Angiopathy and Lobar Intracerebral Hemorrhage. Arch Neurol. 2006;63:148-51.

5. Maat-Schieman M, Roos R, Van Duinen S. Hereditary cerebral hemorrhage with amyloidosis-Dutch type. Neuropathology. 2005;25:288-97.

6. Wermer MJH, Greenberg SM. The growing clinical spectrum of cerebral amyloid angiopathy. Curr Opin Neurol. 2018;31:2835.

7. Bornebroek M, Haan J, Maat-Schieman ML, Van Duinen SG, Roos RA. Hereditary cerebral hemorrhage with amyloidosis-Dutch type (HCHWA-D): I-A review of clinical, radiologic and genetic aspects. Brain Pathol. 1996;6:111-4.

8. Bornebroek M, Haan J, Roos RAC. Hereditary cerebral hemorrhage with amyloidosis-Dutch type (HCHWA-D): A review of the variety in phenotypic expression. Amyloid. 1999;6:215-24.

9. Linn J, Halpin A, Demaerel P, Ruhland J, Giese AD, Dichgans M, van Buchem MA, Bruckmann H, Greenberg SM. Prevalence of superficial siderosis in patients with cerebral amyloid angiopathy. Neurology. 2010;74:1346.

10. Greenberg SM, Charidimou A. Diagnosis of Cerebral Amyloid Angiopathy. Stroke. 2018;49:491-7.

11. Cannistraro RJ, Meschia JF. The Clinical Dilemma of Anticoagulation Use in Patients with Cerebral Amyloid Angiopathy and Atrial Fibrillation. Curr Cardiol Rep. 2018;20:106.

12. Charidimou A, Friedrich JO, Greenberg SM, Viswanathan A. Core cerebrospinal fluid biomarker profile in cerebral amyloid angiopathy: A meta-analysis. Neurology. 2018;90:e754-62.

13. van Etten ES, Verbeek MM, van der Grond J, Zielman R, van Rooden S, van Zwet EW, van Opstal AM, Haan J, Greenberg SM, van Buchem MA, et al. $\beta$-Amyloid in CSF: Biomarker for preclinical cerebral amyloid angiopathy. Neurology. 2017;88:169-76.

14. Verbeek MM, Kremer BPH, Rikkert MO, Van Domburg PHMF, Skehan ME, Greenberg SM. Cerebrospinal fluid amyloid beta(40) is decreased in cerebral amyloid angiopathy. Ann Neurol. 2009;66:245-9. 
15. Zhu X, Xu F, Hoos MD, Lee H, Benveniste H, Nostrand WEV. Reduced Levels of Cerebrospinal Fluid/Plasma Aß40 as an Early Biomarker for Cerebral Amyloid Angiopathy in RTg-DI Rats. Int J Mol Sci. 2020;21:303.

16. Lupu F, Heim DA, Bachmann F, Hurni M, Kakkar VV, Kruithof EKO. Plasminogen Activator Expression in Human Atherosclerotic Lesions. Arterioscler Thromb Vasc Biol. 1995;15:1444-55.

17. Blasi F, Carmeliet P. uPAR: a versatile signalling orchestrator. Nat Rev Mol Cell Biol. 2002;3:932-43.

18. Wakui S, Furusato M, Muto T, Ohshige H, Takahashi H, Ushigome S. Transforming Growth Factor- $\beta$ and Urokinase Plasminogen Activator Presents at Endothelial Cell-Pericyte Interdigitation in Human Granulation Tissue. Microvasc Res. 1997;54:262-9.

19. Mutimer CA, Keragala CB, Markus HS, Werring DJ, Cloud GC, Medcalf RL. Cerebral Amyloid Angiopathy and the Fibrinolytic System: Is Plasmin a Therapeutic Target? Stroke 2021, 52:2707-2714.

20. Tucker HM, Kihiko M, Caldwell JN, Wright S, Kawarabayashi T, Price D, Walker D, Scheff S, McGillis JP, Rydel RE, Estus S. The plasmin system is induced by and degrades amyloid-beta aggregates. J Neurosci. 2000;20:3937-46.

21. Verbeek MM, Van Nostrand WE, Otte-Holler I, Wesseling P, De Waal RM. Amyloid-beta-induced degeneration of human brain pericytes is dependent on the apolipoprotein E genotype. Ann N Y Acad Sci. 2000;903:187-99.

22. Wilhelmus MMM, Otte-Höller I, Davis J, Van Nostrand WE, de Waal RMW, Verbeek MM. Apolipoprotein E Genotype Regulates Amyloid- $\beta$ Cytotoxicity. The Journal of Neuroscience. 2005;25:3621-7.

23. Davis J, Wagner MR, Zhang W, Xu F, Van Nostrand WE. Amyloid $\beta$-Protein Stimulates the Expression of Urokinase-type Plasminogen Activator (UPA) and Its Receptor (UPAR) in Human Cerebrovascular Smooth Muscle Cells. J Biol Chem. 2003;278:19054-61.

24. Miners JS, Baig S, Palmer J, Palmer LE, Kehoe PG, Love S. Abeta-degrading enzymes in Alzheimer's disease. Brain Pathol. 2008;18:240-52.

25. Van Nostrand WE, Porter M. Plasmin cleavage of the amyloid beta-protein: alteration of secondary structure and stimulation of tissue plasminogen activator activity. Biochemistry. 1999;38:11570-6.

26. Davis J, Xu F, Hatfield J, Lee H, Hoos MD, Popescu D, Crooks E, Kim R, Smith SO, Robinson JK, et al. A Novel Transgenic Rat Model of Robust Cerebral Microvascular Amyloid with Prominent Vasculopathy. Am J Pathol. 2018;188:2877-89.

27. Whelan CD, Mattsson N, Nagle MW, Vijayaraghavan S, Hyde C, Janelidze S, Stomrud E, Lee J, Fitz L, Samad TA, et al. Multiplex proteomics identifies novel CSF and plasma biomarkers of early Alzheimer's disease. Acta Neuropathol Commun. 2019;7:169-9.

28. Martorana A, Sancesario GM, Esposito Z, Nuccetelli M, Sorge R, Formosa A, Dinallo V, Bernardi G, Bernardini S, Sancesario G. Plasmin system of Alzheimer's disease patients: CSF analysis. J Neural Transm (Vienna). 2012;119:763-9.

29. Hanzel CE, lulita MF, Eyjolfsdottir H, Hjorth E, Schultzberg M, Eriksdotter M, Cuello AC. Analysis of Matrix Metallo-Proteases and the Plasminogen System in Mild Cognitive Impairment and Alzheimer's Disease Cerebrospinal Fluid. J Alzheimers Dis. 2014;40:667-78.

30. Camoin L, Pannell R, Anfosso F, Lefevre JP, Sampol J, Gurewich V, Dignat-George F. Evidence for the expression of urokinasetype plasminogen activator by human venous endothelial cells in vivo. Thromb Haemost. 1998;80:961-7.

31. Dosne AM, Dupuy E, Bodevin E. Production of a fibrinolytic inhibitor by cultured endothelial cells derived from human umbilical vein. Thromb Res. 1978;12:377-87.

32. Reuning U, Sperl S, Kopitz C, Kessler H, Krüger A, Schmitt M, Magdolen V. Urokinase-type plasminogen activator (UPA) and its receptor (UPAR): development of antagonists of UPA/UPAR interaction and their effects in vitro and in vivo. Curr Pharm Des. 2003;9:1529-43.

33. Lee J-Y, Kweon H-S, Cho E, Lee J-Y, Byun H-R, Kim DH, Kim Y-H, Han P-L, Koh J-Y. Upregulation of tPA/plasminogen proteolytic system in the periphery of amyloid deposits in the Tg2576 mouse model of Alzheimer's disease. Neurosci Lett. 2007;423:827.

34. Tucker HM, Kihiko-Ehmann M, Wright S, Rydel RE, Estus S. Tissue plasminogen activator requires plasminogen to modulate amyloid-beta neurotoxicity and deposition. J Neurochem. 2000;75:2172-7.

Page $12 / 16$ 
35. Melchor JP, Pawlak R, Strickland S. The tissue plasminogen activator-plasminogen proteolytic cascade accelerates amyloidbeta (Abeta) degradation and inhibits Abeta-induced neurodegeneration. J Neurosci. 2003;23:8867-71.

36. Akhter H, Huang WT, van Groen T, Kuo HC, Miyata T, Liu RM. A Small Molecule Inhibitor of Plasminogen Activator Inhibitor-1 Reduces Brain Amyloid- $\beta$ Load and Improves Memory in an Animal Model of Alzheimer's Disease. J Alzheimers Dis. 2018;64:447-57.

37. Asahina M, Yoshiyama Y, Hattori T. Expression of matrix metalloproteinase-9 and urinary-type plasminogen activator in Alzheimer's disease brain. Clin Neuropathol. 2001;20:60-3.

38. Barker R, Kehoe PG, Love S. Activators and inhibitors of the plasminogen system in Alzheimer's disease. J Cell Mol Med. 2012;16:865-76.

39. Bi Oh S, Suh N, Kim I, Lee JY. Impacts of aging and amyloid- $\beta$ deposition on plasminogen activators and plasminogen activator inhibitor-1 in the Tg2576 mouse model of Alzheimer's disease. Brain Res. 2015;1597:159-67.

40. Diaz A, Merino P, Guo J-D, Yepes MA, McCann P, Katta T, Tong EM, Torre E, Rangaraju S, Yepes M. Urokinase-type Plasminogen Activator Protects Cerebral Cortical Neurons From Soluble Aß-induced Synaptic Damage. The Journal of Neuroscience 2020:JN-RM-2804-2819.

41. Kutz SM, Higgins CE, Higgins PJ. Novel Combinatorial Therapeutic Targeting of PAl-1 (SERPINE1) Gene Expression in Alzheimer's Disease. Mol Med Ther. 2012;1:106.

42. Liu RM, van Groen T, Katre A, Cao D, Kadisha I, Ballinger C, Wang L, Carroll SL, Li L. Knockout of plasminogen activator inhibitor 1 gene reduces amyloid beta peptide burden in a mouse model of Alzheimer's disease. Neurobiol Aging.

2011;32:1079-89.

43. Wang DS, Dickson DW, Malter JS. beta-Amyloid degradation and Alzheimer's disease. J Biomed Biotechnol. 2006;2006:58406.

44. Murphy G, Atkinson S, Ward R, Gavrilovic J, Reynolds JJ. The role of plasminogen activators in the regulation of connective tissue metalloproteinases. Ann N Y Acad Sci. 1992;667:1-12.

45. Carmeliet P, Moons L, Lijnen R, Baes M, Lemaître V, Tipping P, Drew A, Eeckhout Y, Shapiro S, Lupu F, Collen D. Urokinasegenerated plasmin activates matrix metalloproteinases during aneurysm formation. Nat Genet. 1997;17:439-44.

46. Mahmood N, Mihalcioiu C, Rabbani SA. Multifaceted Role of the Urokinase-Type Plasminogen Activator (UPA) and Its Receptor (UPAR): Diagnostic, Prognostic, and Therapeutic Applications. Frontiers in Oncology 2018, 8.

47. Mazzieri R, Masiero L, Zanetta L, Monea S, Onisto M, Garbisa S, Mignatti P. Control of type IV collagenase activity by components of the urokinase-plasmin system: a regulatory mechanism with cell-bound reactants. The EMBO Journal. 1997;16:2319-32.

48. Martínez-Lizana E, Carmona-Iragui M, Alcolea D, Gómez-Choco M, Vilaplana E, Sánchez-Saudinós MB, Clarimón J, HernándezGuillamon M, Munuera J, Gelpi E, et al. Cerebral amyloid angiopathy-related atraumatic convexal subarachnoid hemorrhage: an ARIA before the tsunami. J Cereb Blood Flow Metab. 2015;35:710-7.

49. van Etten ES, Verbeek MM, van der Grond J, Zielman R, van Rooden S, van Zwet EW, van Opstal AM, Haan J, Greenberg SM, van Buchem MA, et al. $\beta$-Amyloid in CSF: Biomarker for preclinical cerebral amyloid angiopathy. Neurology. 2017;88:169-76.

50. Kuiperij HB, Hondius DC, Kersten I, Versleijen AAM, Rozemuller AJM, Greenberg SM, Schreuder F, Klijn CJM, Verbeek MM. Apolipoprotein D: a potential biomarker for cerebral amyloid angiopathy. Neuropathol Appl Neurobiol 2019.

\section{Figures}



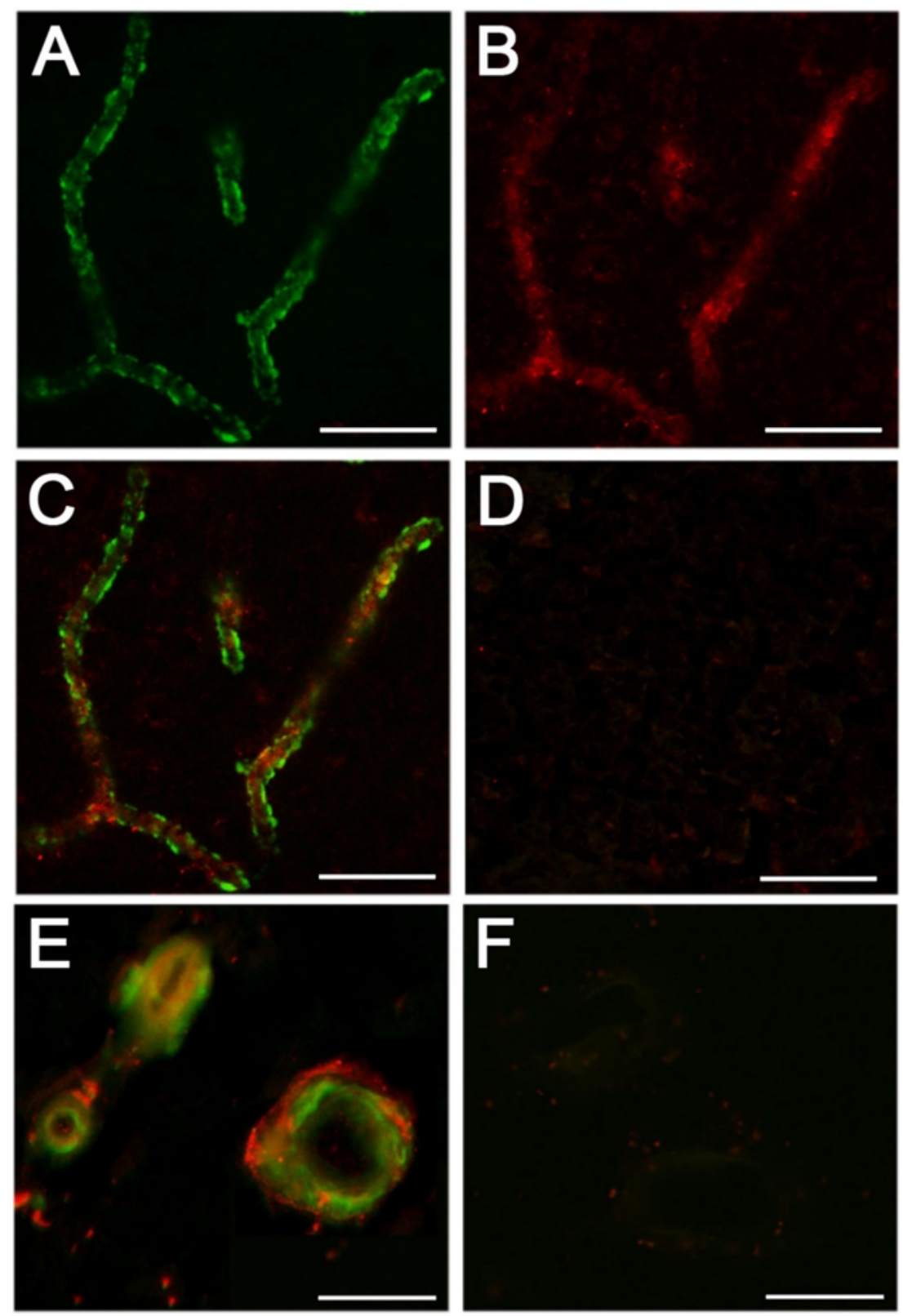

\section{Figure 1}

Cerebral vascular A $\beta$ deposition and uPA expression in rTg-DI rats, WT rats, human CAA patients and control subjects. Twelve months old rTg-DI rat brain sections were stained for (A) fibrillar amyloid using thioflavin S and (B) immunolabeled for uPA. (C) Composite image showing co-localization of thioflavin $S+$ UPA in rTg-DI models. (D) Composite image showing absence of A $\beta$ deposits and UPA expression in a WT model. (E) Composite image showing co-localization of thioflavin S + UPA in a human sporadic CAA patient. (F) Composite image showing absence of $A \beta$ deposits and uPA expression in a human control subject. Scale bars $=50 \mu \mathrm{m}$ and $A \beta$ in green, uPA in red. 

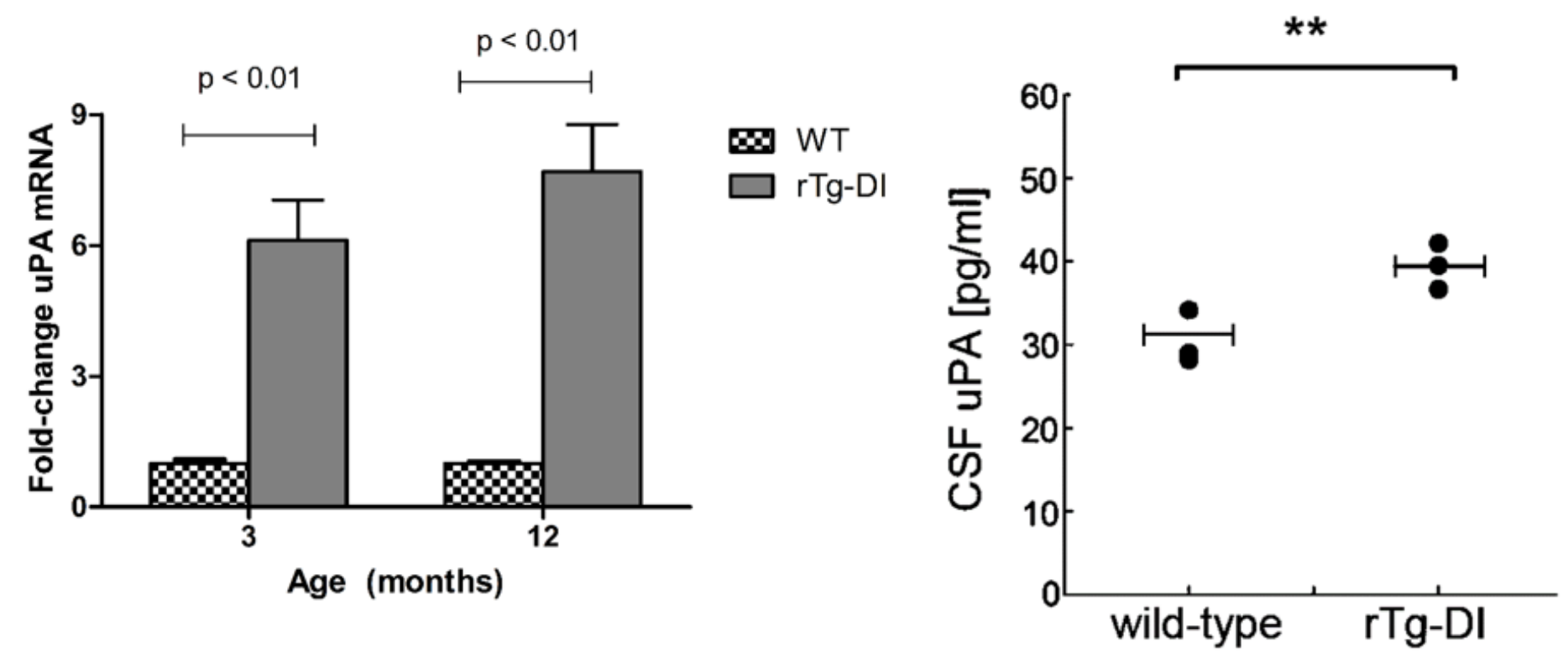

Figure 2

Elevated uPA expression in brain tissue and CSF of rTg-DI rats. (A) mRNA expression of PLAU gene was assessed by qPCR in $W T / r T g-D I$ rats at $3(n=4 / 7)$ and 12 months $(n=5 / 5)$. Expression was significantly elevated at each age in $r T g$-DI rats compared with WT rats (T-test; $p<0.01)$. (B) CSF uPA levels were assessed using a rat uPA ELISA in rTg-DI and WT rats at 3 months of age. uPA levels were significantly elevated in rTg-DI rats (T-test, $n=6, p=0.03$ ).
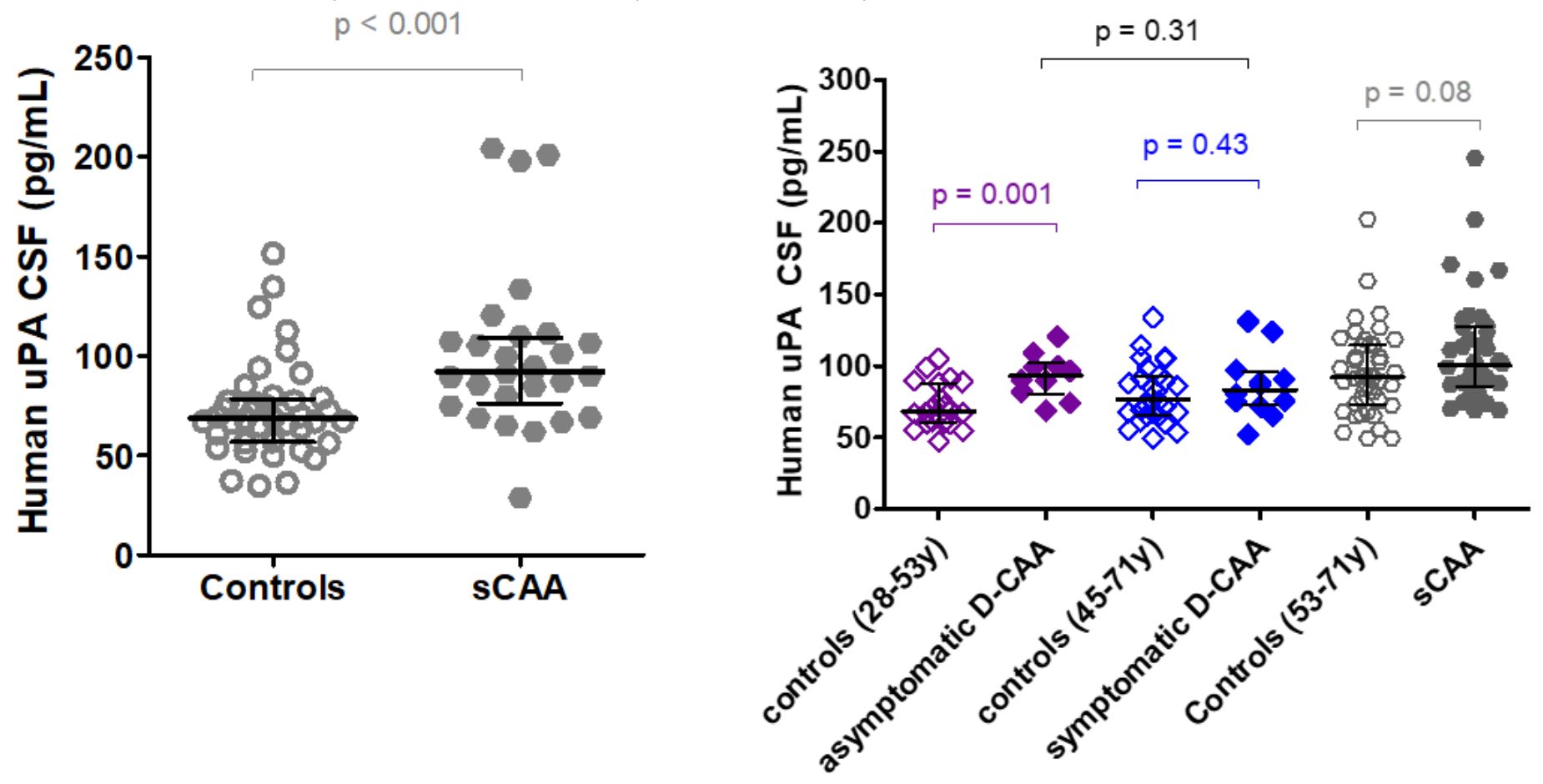

Figure 3

Comparison of human CSF uPA levels in CAA patient and control groups. Scatter plots of CSF uPA levels in our (A) discovery and (B) validation studies. In our discovery study, uPA levels were significantly elevated in sCAA (in dark grey; $n=27$ ) compared to control subjects (in light grey; $n=40)$ ( $M U p<0.001$ ), even after adjusting for age ( $M U p=0.05)$. In our validation studies, uPA levels in sCAA (in dark grey; $n=38$ ) were borderline non-significant ( $M U p=0.08)$, but significantly elevated after adjusting for age (MU $p=0.03$ ) compared to controls (in light grey; $n=40$ ). Asymptomatic $D-C A A$ patients (in dark purple; $n=11$ ) presented elevated uPA 
levels in CSF compared to their respective controls (in light purple; $n=22 ; M U p=0.01$; after adjusting for age, $M U p=0.09$ ), whereas symptomatic $D$-CAA patients (in dark blue; $n=12$ ) did not show an elevation ( $M U p=0.46$; after adjusting for age, $M U p=0.44$ ) compared to age-matched controls (in light blue; $n=28$ ). All data presented as median with IQR. 